\title{
Accelerating Feldman-Cousins for NOvA using NERSC Supercomputers
}

\section{Derek Doyle}

This document was prepared by [NOvA Collaboration] using the resources of the Fermi National Accelerator Laboratory (Fermilab), a U.S. Department of Energy, Office of Science, HEP User Facility. Fermilab is managed by Fermi Research Alliance, LLC (FRA), acting under Contract No. DE-AC02-07CH11359. 


\section{Measuring Oscillation Parameters}
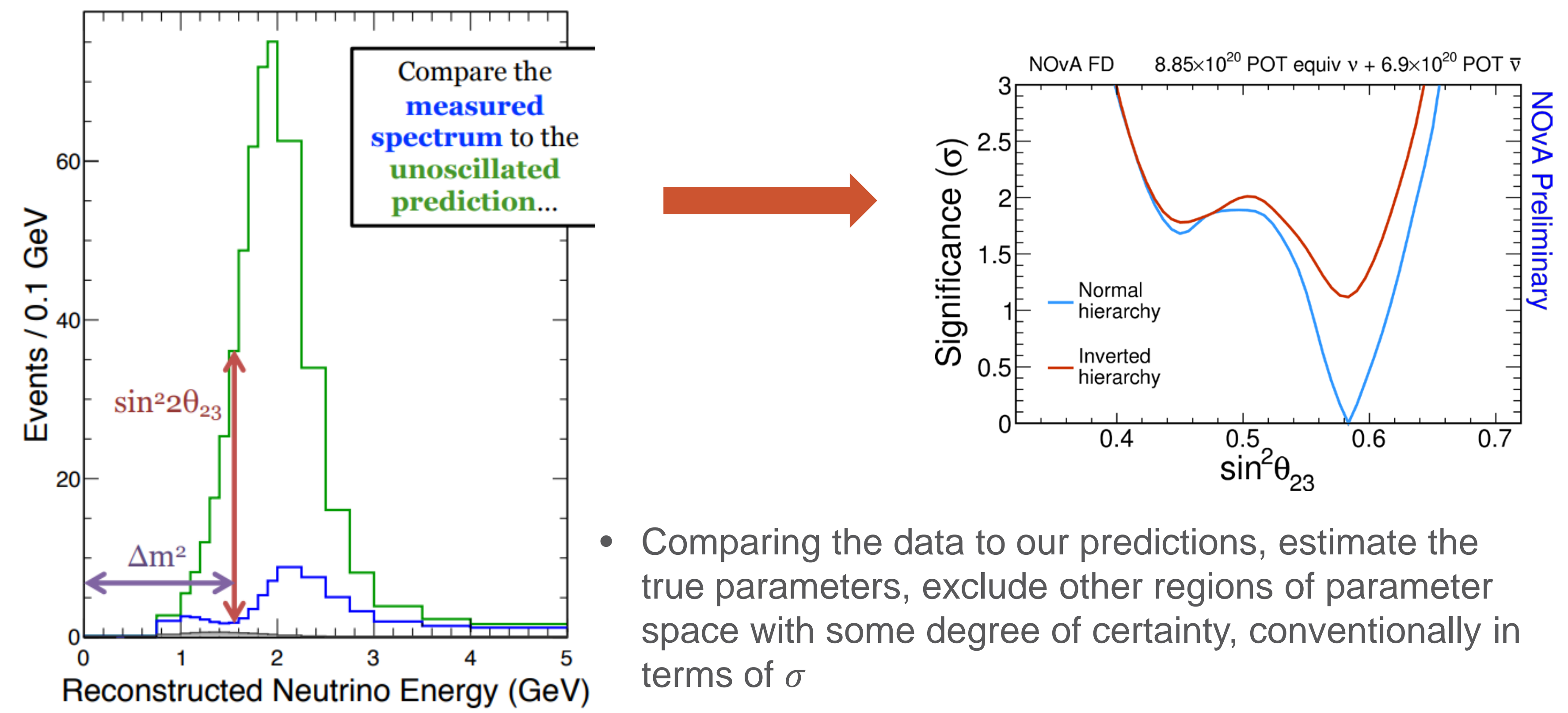

- Comparing the data to our predictions, estimate the true parameters, exclude other regions of parameter space with some degree of certainty, conventionally in terms of $\sigma$ 


\section{Parameter Estimation}

- Goal: Determine the likelihood that $\vec{\theta}$ are the true parameters

- Find $\vec{\theta}$ that maximize the Likelihood function: $\mathcal{L}\left(\vec{\theta}_{\text {best }}\right)$

$$
\chi^{2}(\vec{\theta}) \equiv-2 \log \mathcal{L}(\vec{\theta})=2 \sum_{i}^{\text {bins }}\left[e_{i}(\vec{\theta})-o_{i}+o_{i} \log \frac{o_{i}}{e_{i}(\vec{\theta})}\right] \begin{aligned}
& \overrightarrow{\boldsymbol{\theta}}: \text { Set of Oscillation parameters } \\
& \boldsymbol{o}_{i} \text { : Observed data } \\
& \boldsymbol{e}_{\boldsymbol{i}}(\overrightarrow{\boldsymbol{\theta}}): \text { Expected observation }
\end{aligned}
$$

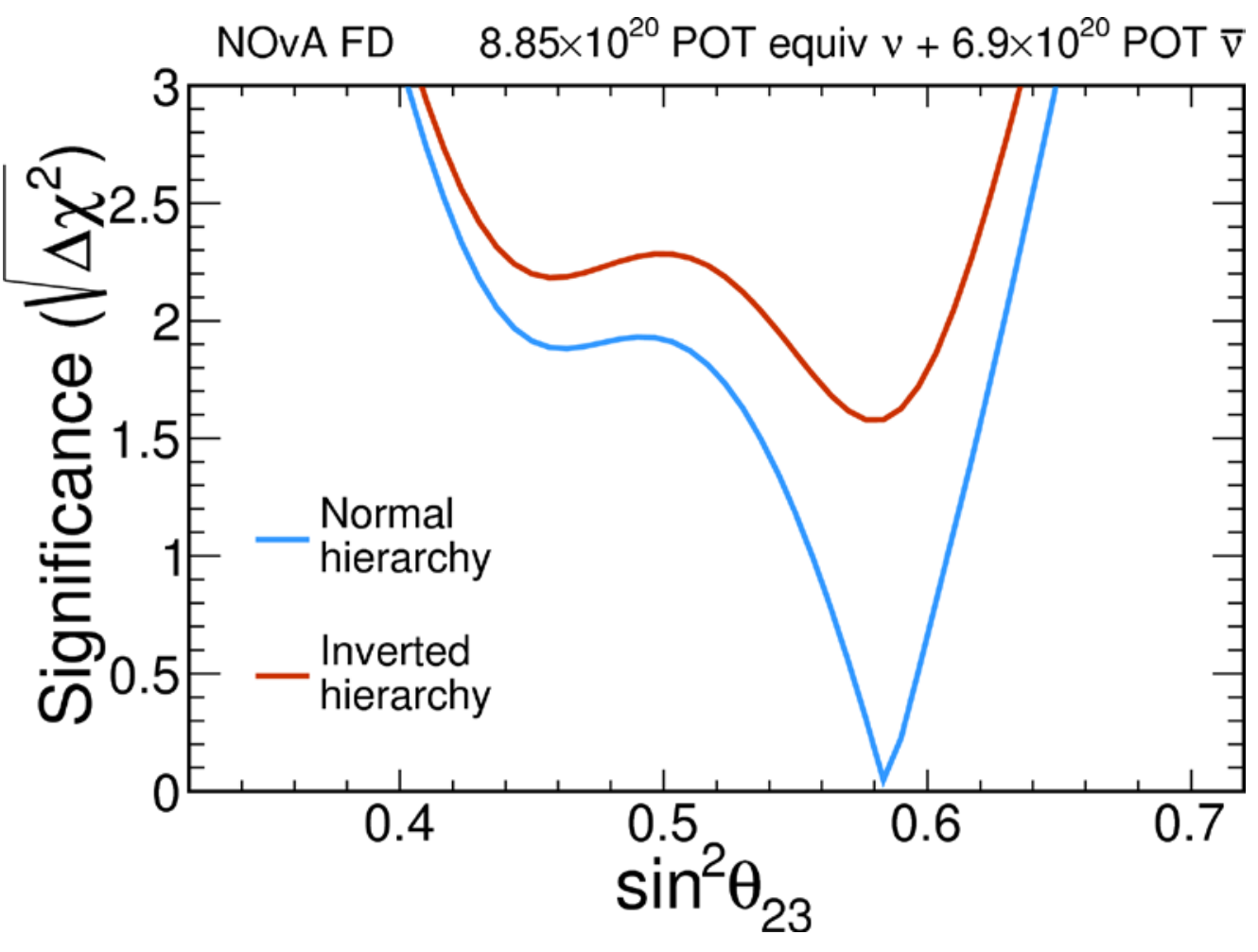

- To measure the significance with which we exclude other parameters, $\vec{\theta}$, as the true parameters, we calculate $\chi^{2}(\vec{\theta})$ and construct the test statistic, $\Delta \chi^{2}=\chi^{2}(\vec{\theta})-\chi^{2}\left(\vec{\theta}_{\text {best }}\right)$

- Conveniently, this distribution converges to a $\chi^{2}$ distribution under certain assumption

- $\quad$ Moreover, under these assumptions, $\sigma=\sqrt{\Delta \chi^{2}}$ 


\section{$\chi^{2}$ Distribution}
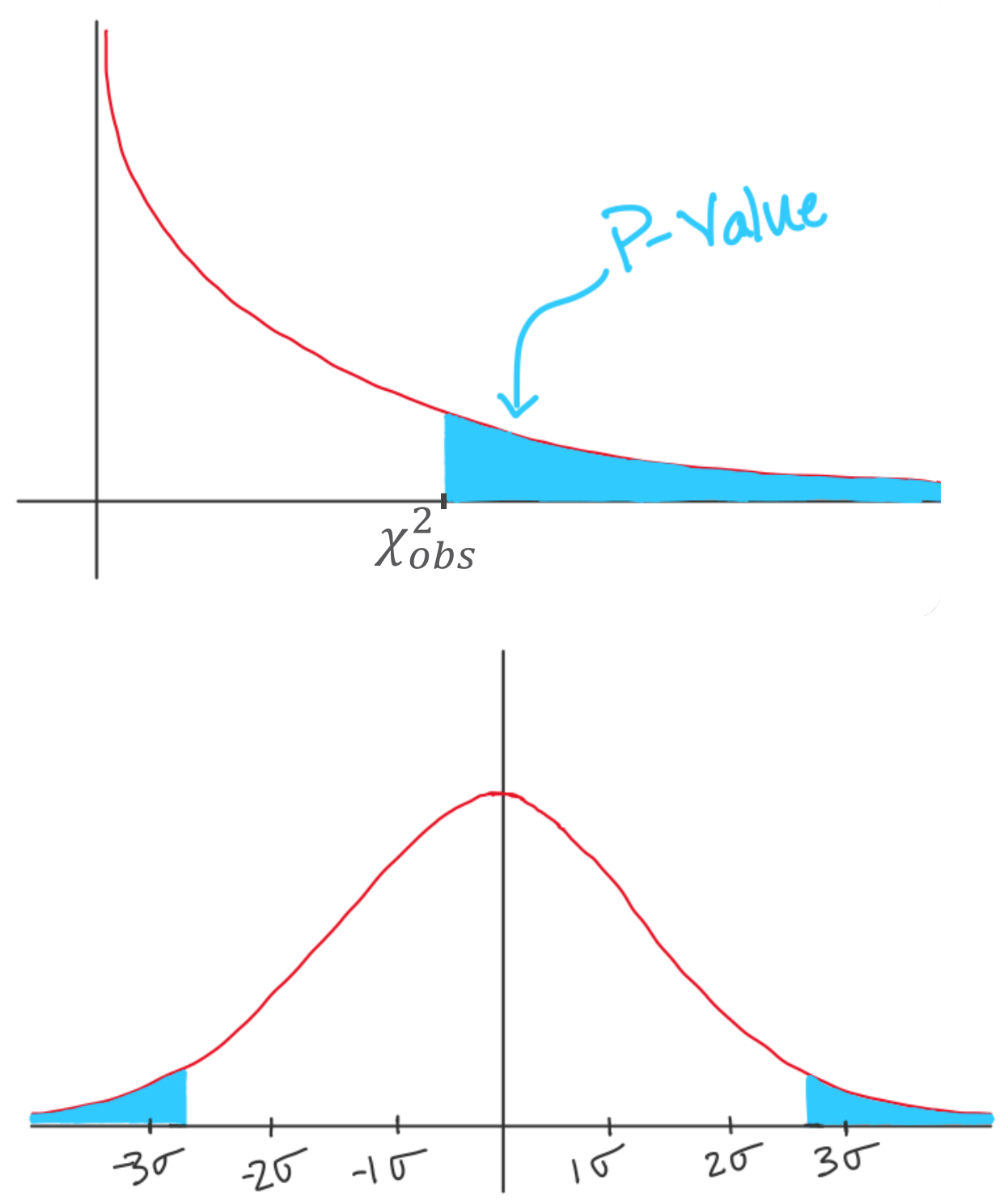

- Often used for determining goodness of fit

- Repeating experiment may result in different observations

- Want to know the probability of finding a $\chi^{2}$

- P-Value: probability of finding a $\chi^{2}$ at least as large as than the one you measure

- Tells you how probable it was to measure a $\chi^{2}$ by accident

$$
p \text {-value }=\int_{\chi_{o b s}^{2}}^{\infty} f(\chi) d \chi
$$

- Critical Value: value of test statistic corresponding to a given $\mathrm{p}$-value

- Report a significance in terms of the critical value of a standard normal distribution corresponding to that $p$ value 


\section{Case Study: 1D Profile}

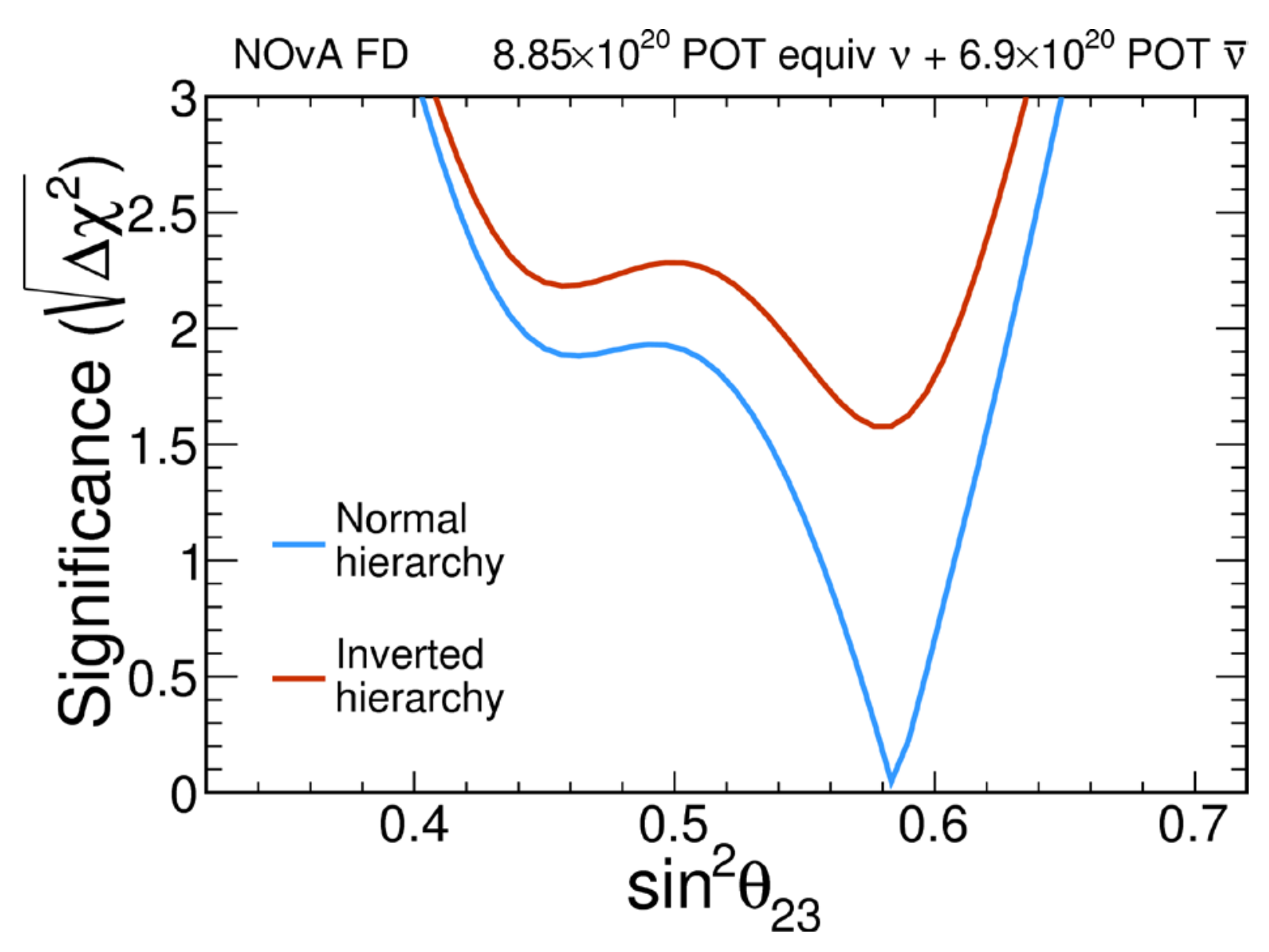

- At each point in parameter space, calculate a $\Delta \chi^{2}(\vec{\theta})$

$$
\Delta \chi^{2}=\chi^{2}(\vec{\theta})-\chi^{2}\left(\vec{\theta}_{\text {best }}\right)
$$

- We end up with the trajectory that minimizes $\Delta \chi^{2}$ along one axis

- Profile over other parameters

- Can we use a standard $\chi^{2}$ ? 


\section{Wilks' theorem}

- A $\Delta \chi^{2}$ distribution converges to a standard $\chi^{2}$ if two conditions are met:

1. Large Statistics

2. Parameters are sufficiently far from physical boundaries

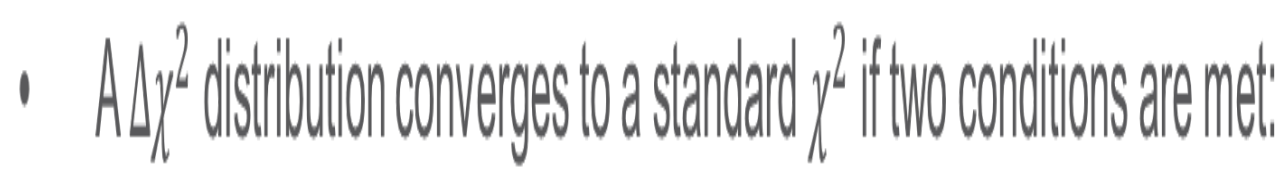

1. Latge Statisitics

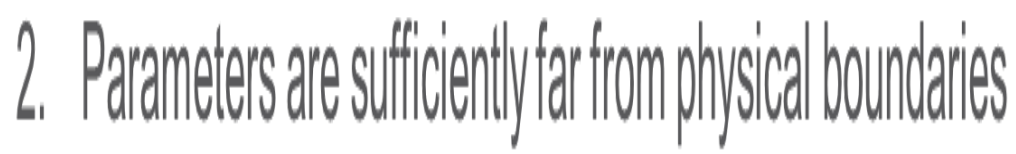




\section{Wilks' theorem}

\begin{tabular}{lc}
\hline Total $\overline{\boldsymbol{v}}_{\boldsymbol{e}}$ Observed & $\mathbf{1 8}$ \\
\hline Total Prediction & 15.9 \\
\hline Wrong-sign & 1.1 \\
Beam Bkgd. & 3.5 \\
Cosmic Bkgd. & 0.7 \\
Total Bkgd. & 5.3 \\
\hline
\end{tabular}
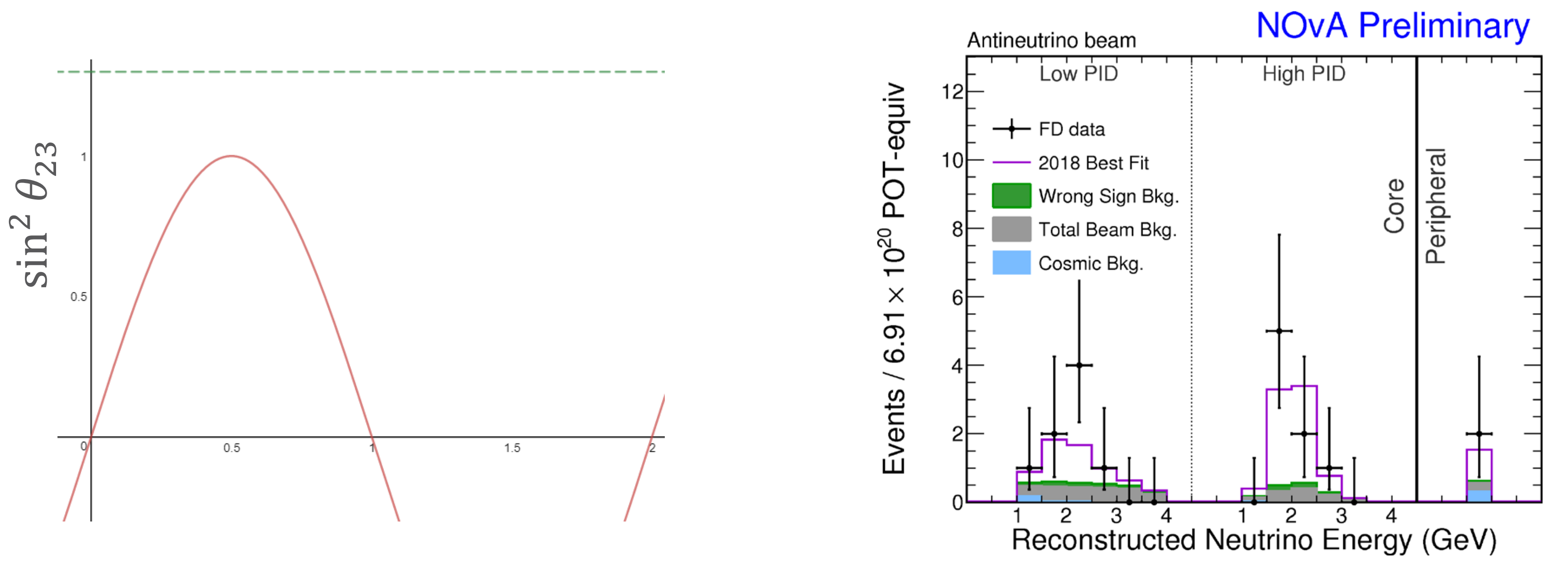


\section{Feldman-Cousins Procedure}

- Wilks' result is a convenience if his conditions are met

- If we could find a true $\Delta \chi^{2}$ distribution, we could calculate a p-value from it just like any other distribution function and quote a true significance

- So let's do that... empirically

- Recall a p-value is like proportion of your test statistic that is distributed beyond a certain value

- So if we can find a similar proportion of an empirical distribution, we can quote a significance

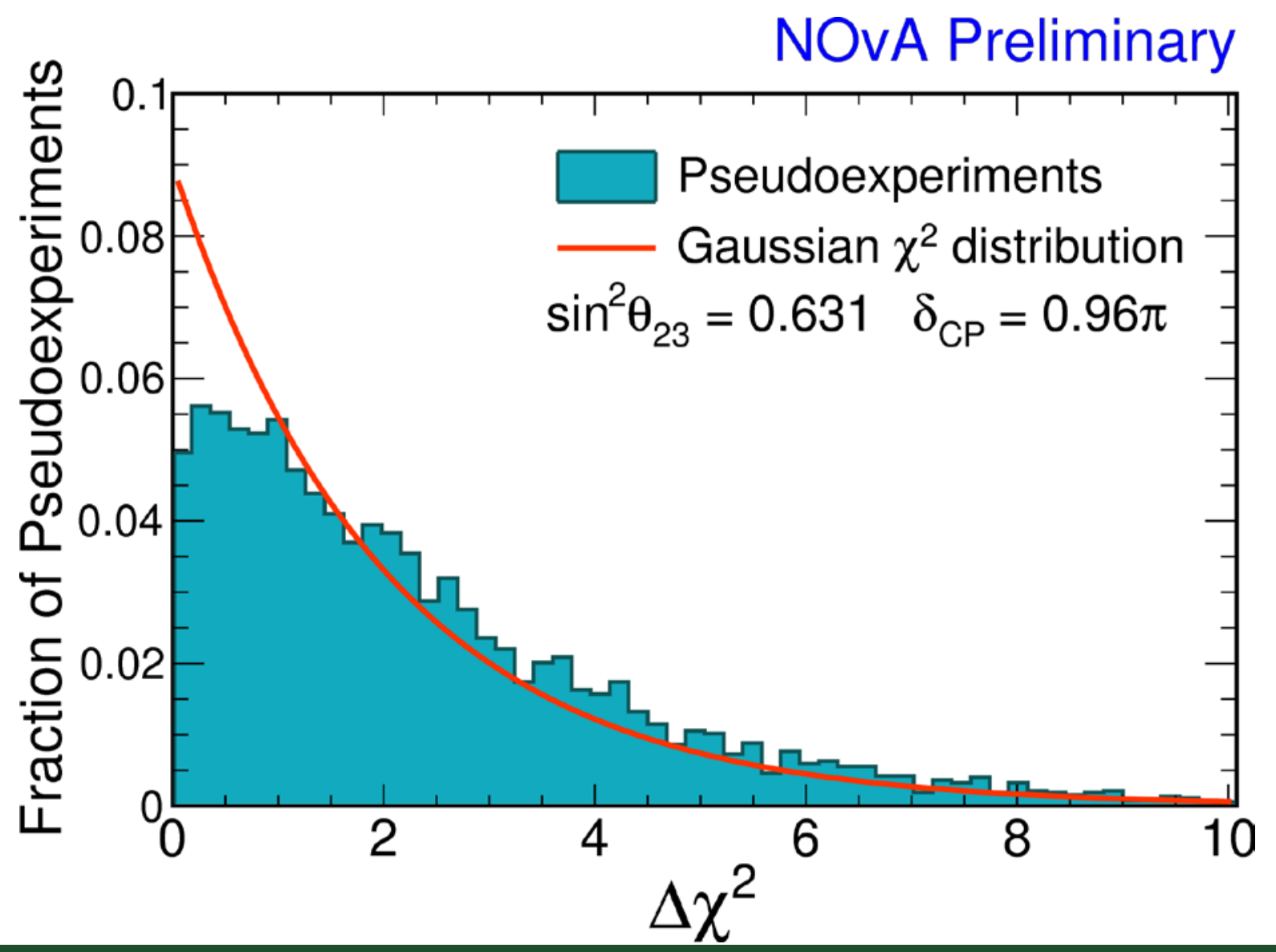




\section{Feldman-Cousins Procedure}

- At a given point in parameter space we want to know, "does our prediction at a set of test parameters, $\vec{\theta}_{\text {test }}$, agree with our data?"

- We need a $\Delta \chi^{2}$ distribution where this is true in order to reject our hypothesis

- i.e, In a world where the true parameters are $\vec{\theta}_{\text {test }}$, given statistical fluctuations, how would the $\Delta \chi^{2}$ test statistic be distributed?

1. Generate data at $\theta_{\text {test }}$ that is Poisson-fluctuated (pseudoexperiment) in order to get value of $\Delta \chi^{2}$

a. Do this many times to build a distribution

2. Find the proportion of pseudoexperiments above observed $\Delta \chi^{2}$

3. Quote a significance based off that $p$-value

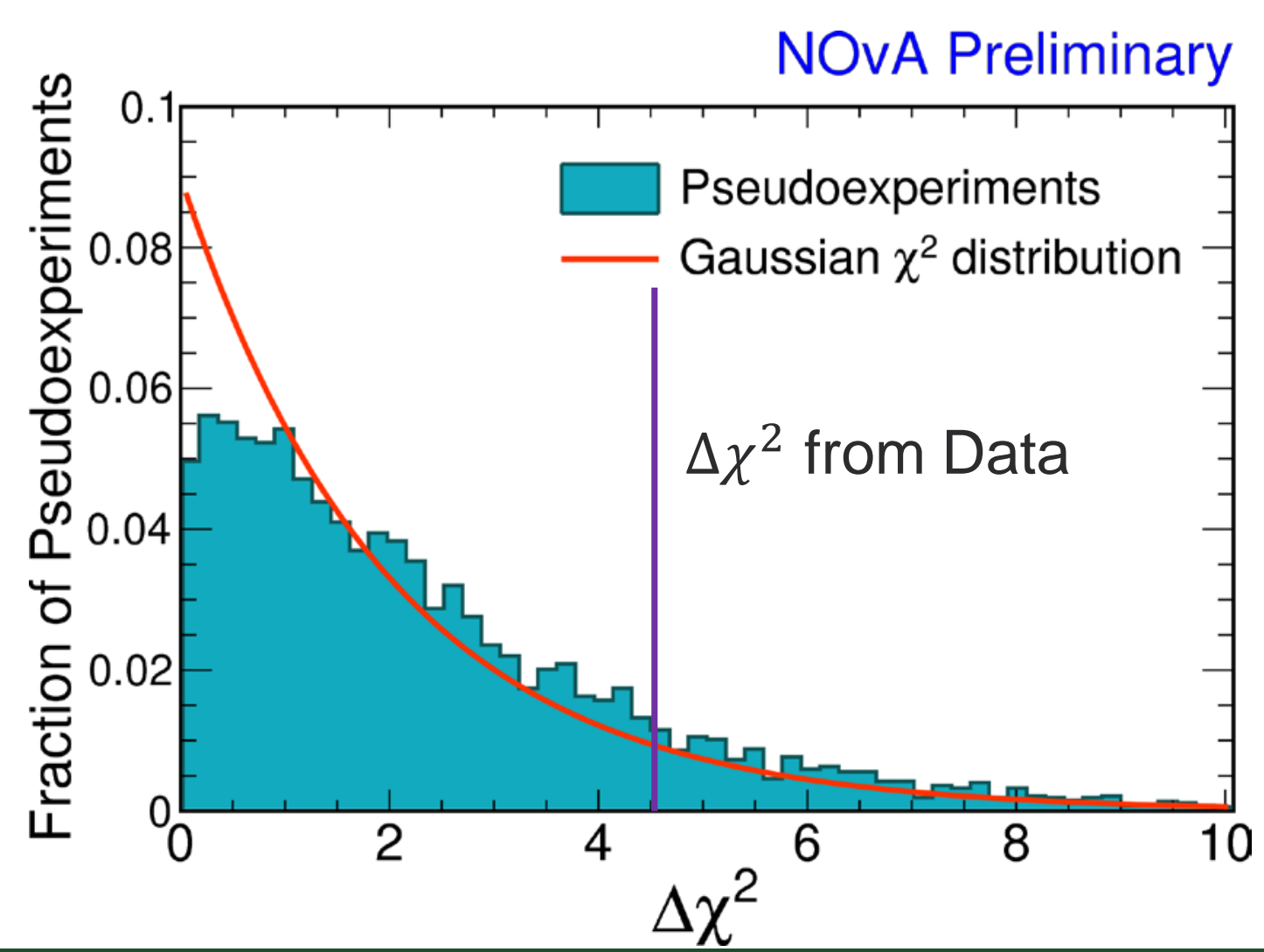




\section{Correcting a contour}

- The same thing can be done in 2D

- Like 1D profiles: Now we find the surface in parameter space that minimizes $\Delta \chi^{2}$

- Draw lines of constant significance ( $p$-value)

Difference between empirical and gaussian $\Delta \chi_{c r i t}^{2}$ for a $90 \%$ Confidence Interval

NOvA Preliminary

- Left-skewed distribution:

- Decreases $\Delta \chi_{\text {crit }}^{2}$

- Increases ability to exclude
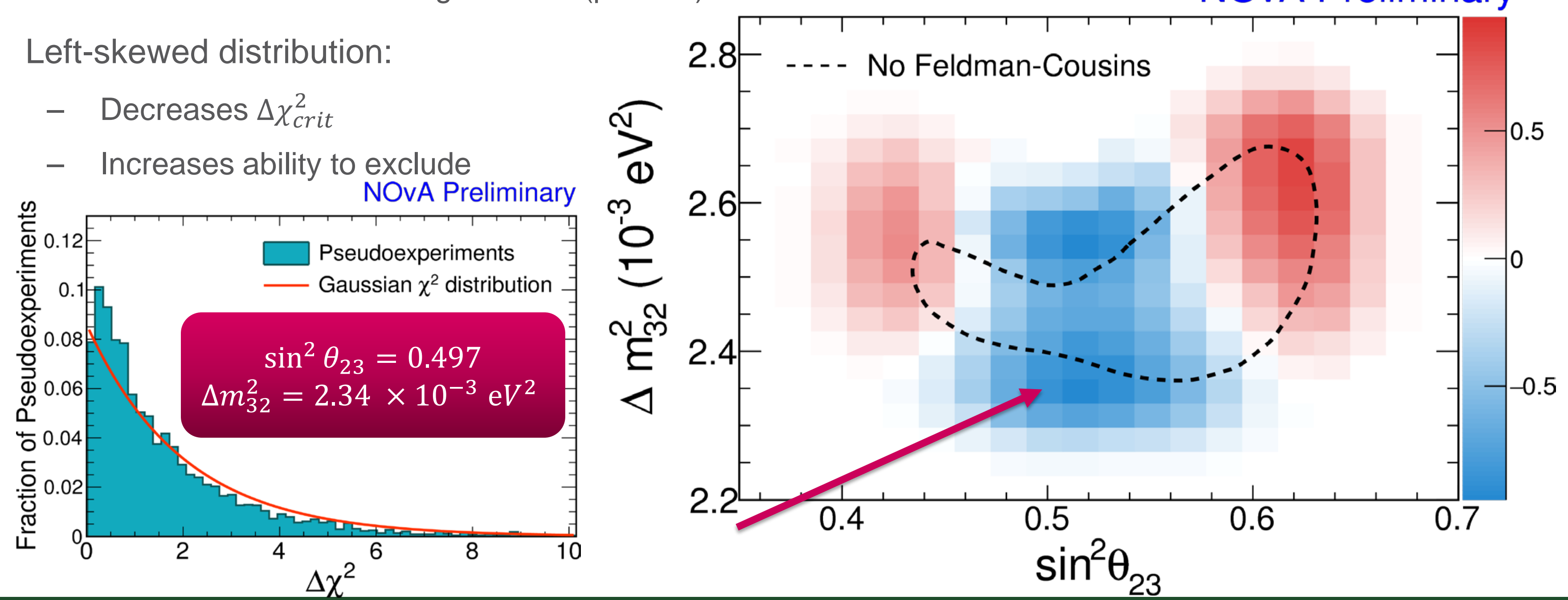


\section{Correcting a contour}

- The same thing can be done in 2D

- Like 1D profiles: Now we find the surface in parameter space that minimizes $\Delta \chi^{2}$

- Draw lines of constant significance ( $p$-value)

Difference between empirical and gaussian $\Delta \chi_{\text {crit }}^{2}$ for a $90 \%$ Confidence Interval

NOvA Preliminary

- Left-skewed distribution:

- Decreases $\Delta \chi_{\text {crit }}^{2}$

- Increases ability to exclude
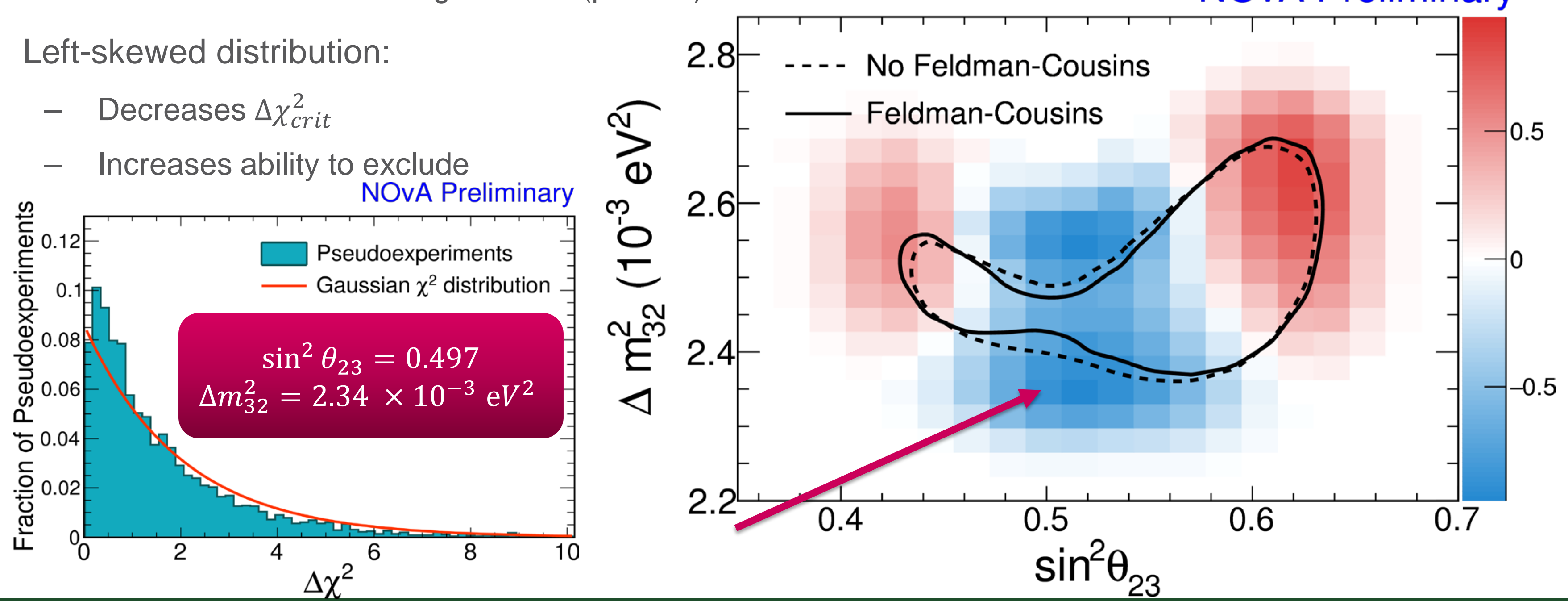


\section{Computational considerations}

- We need to generate $\Delta \chi^{2}$ distributions at each point in parameter space that we're sampling

- Profiles: 1,210 bins

- Contours: 471 bins

- In each bin, we need at least 3,000 pseudoexperiments in $\Delta \chi^{2}$ to generate an accurate empirical distribution

- In previous years, this has been done on the FermiGrid with results in $\sim 4$ weeks

- With the addition of antineutrino data and a longer list of systematics, the FermiGrid was no longer an option

\begin{tabular}{ll} 
Required No. Bins & $\begin{array}{l}\text { Required No. } \\
\text { Pseudoexperiments }\end{array}$ \\
\hline 1,681 & $6,724,000$ \\
\hline
\end{tabular}




\section{NERSC - Lawrence Berkeley National Lab}

(National Energy Research Scientific Computing)

$$
\text { CORI - Phase I }
$$

- Intel "Haswell" at $2.3 \mathrm{GHz}$

- Total Cores: 76,416

- Capable of 2.81 PFlops

- 32 cores share 128 GB memory

- Each core can support 2 FC jobs
CORI - Phase II

- Intel "Knights Landing" (KNL) at $1.4 \mathrm{GHz}$

- Total Cores: 658,784

- Capable of 29.5 PFlops

- 68 cores share 96 GB memory

- Each core can support 1 FC job

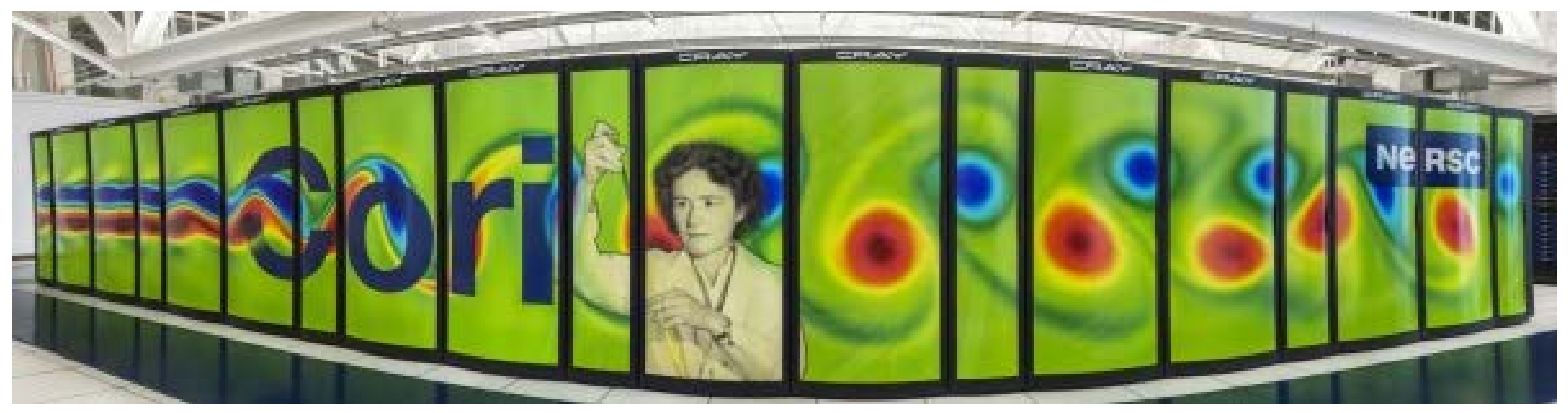




\section{The NERSC Advantage}

\section{First Run}

- Peaked over 1 million cores

- Largest Condor pool ever!

- Noticed the fits were misbehaving due to added complexity

- NERSC allowed us to correct for this unforeseen complication and quickly turn around results

\section{Second Run}

- Peaked $\sim 700,000$ cores

- Second Largest Condor pool ever!

- Over 9 million total points

- 36 hours
NOvA Claimed CPUs

\section{2}




\section{The NERSC Advantage}

\section{$2017 v$ Analysis*}

- $\quad$ Fitting 5 histograms

- 4 profiles

- 4 contours

- $\quad \sim 4$ weeks to completion (FermiGrid)

- 2 minutes per $\Delta \chi^{2}$ (NERSC)

- Reproduced on NERSC in a few hours

\section{$2018 \bar{v}+v$ Analysis}

- Fitting 10 histograms

- 10 profiles

- 4 contours

- 36 hours to completion (NERSC)

- 10 minutes per $\Delta \chi^{2}$

- 36 hours to completion (NERSC)

- $\sim 5$ months on FermiGrid 


\section{Results and Conclusions}

- Feldman-Cousins Corrections have a large effect on our results

- Low statistics and physical boundaries require an empirical approach to stating a significance

- Part of a larger project: HEP Data Analytics on HPC

- Pythia8 and detector simulation tuning for LHC experiments

- Improve performance and precision of neutrino cross-section analyses

- We hope to develop tools made available to all members of the HEP community in achieving similar advances

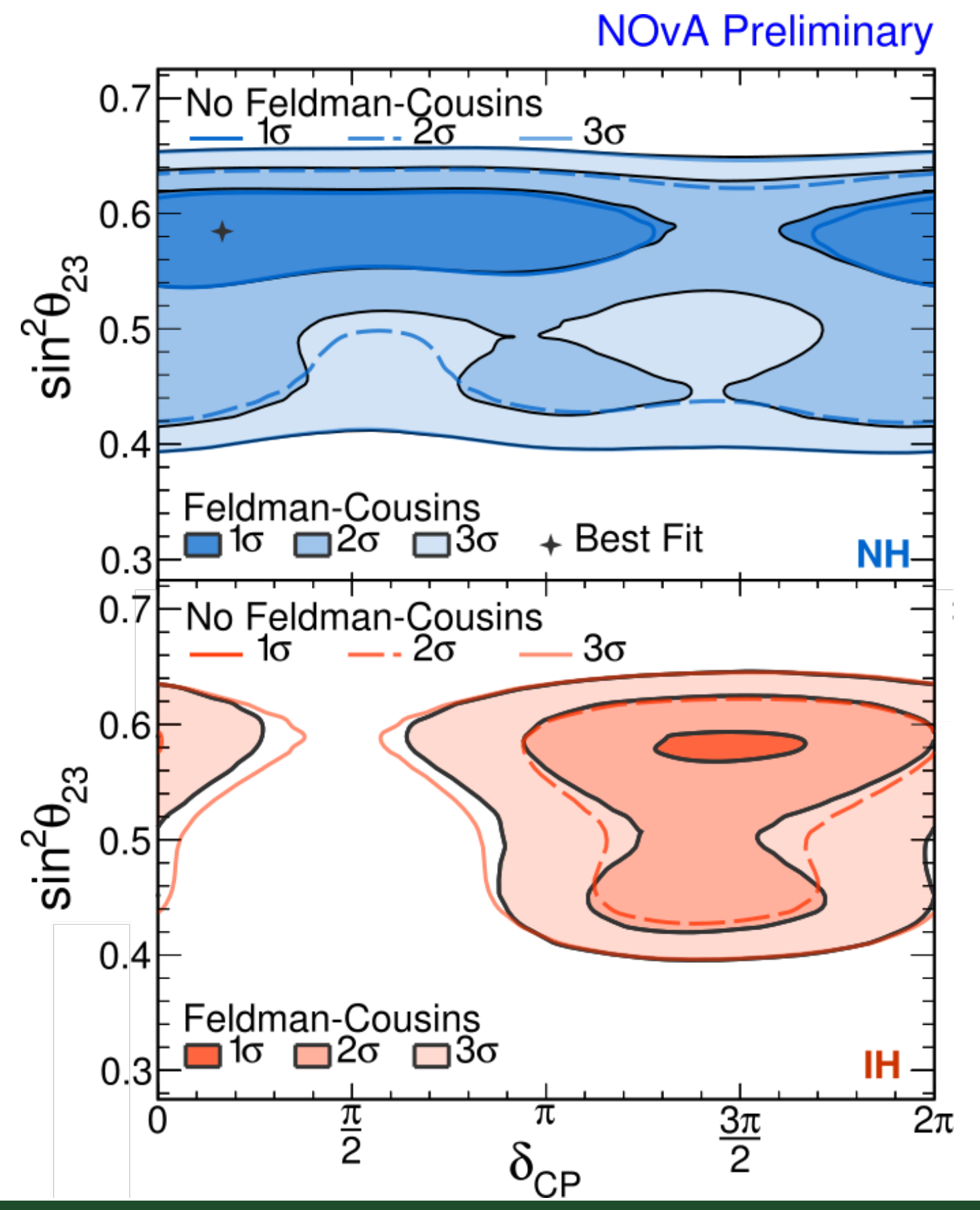




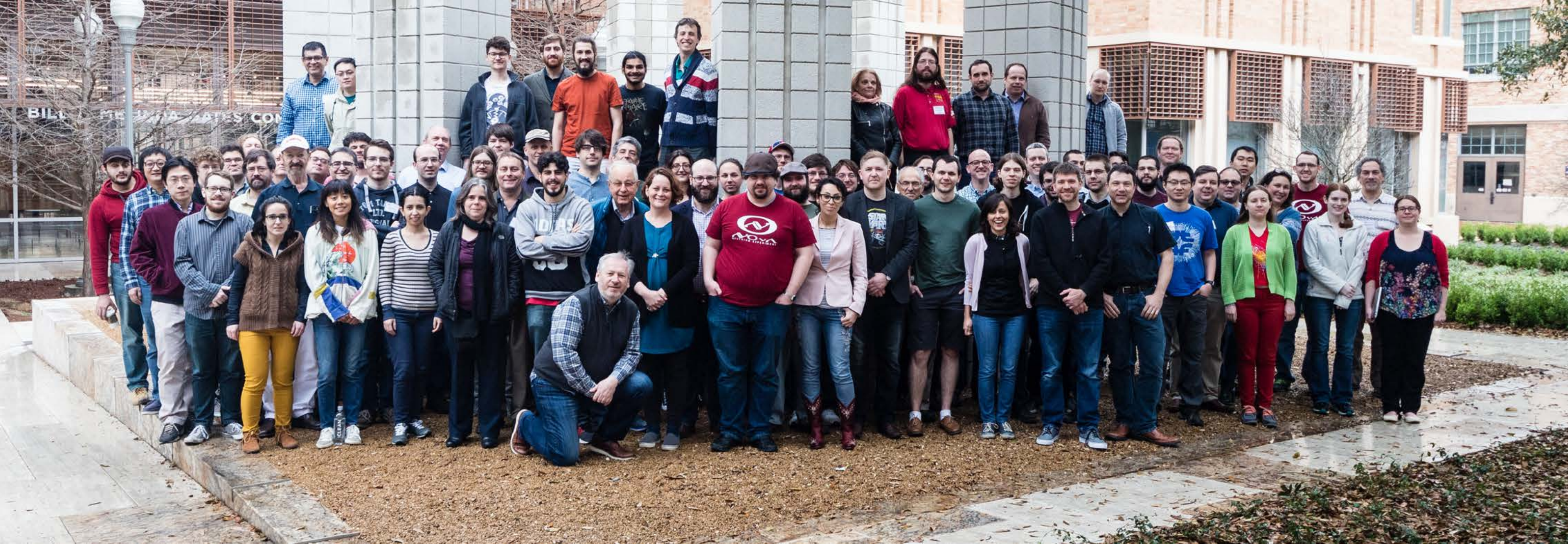

Thank you

ColoradoState University

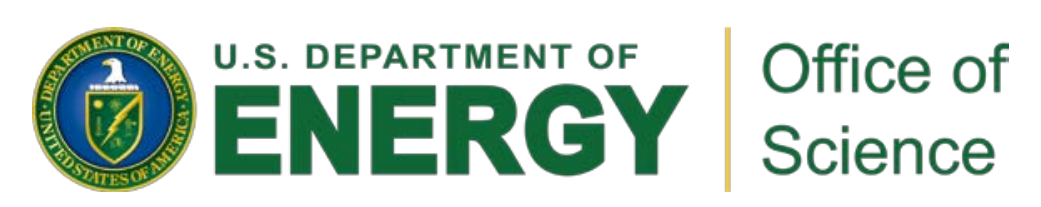

Nerse

National Energy Research
Scientific Computing Center

Scientific Computing Center

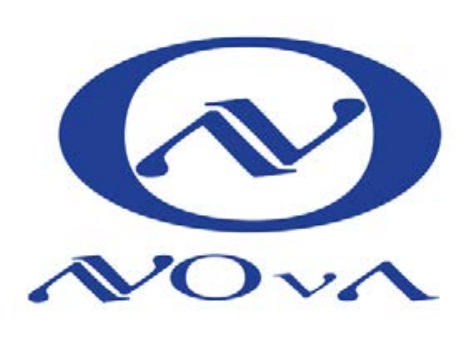




\section{How we run on CORI}

- Docker Images: Like a Virtual Machine, but has a direct line to the machine's kernel

- What is in our image:

- Slf6.7

- NOvASoft

- $\quad$ Spectra/inputs to the FC root macro

- Jobs submitted to CORI mount the image

- Each job can run a separate image instance, called a container, on available threads
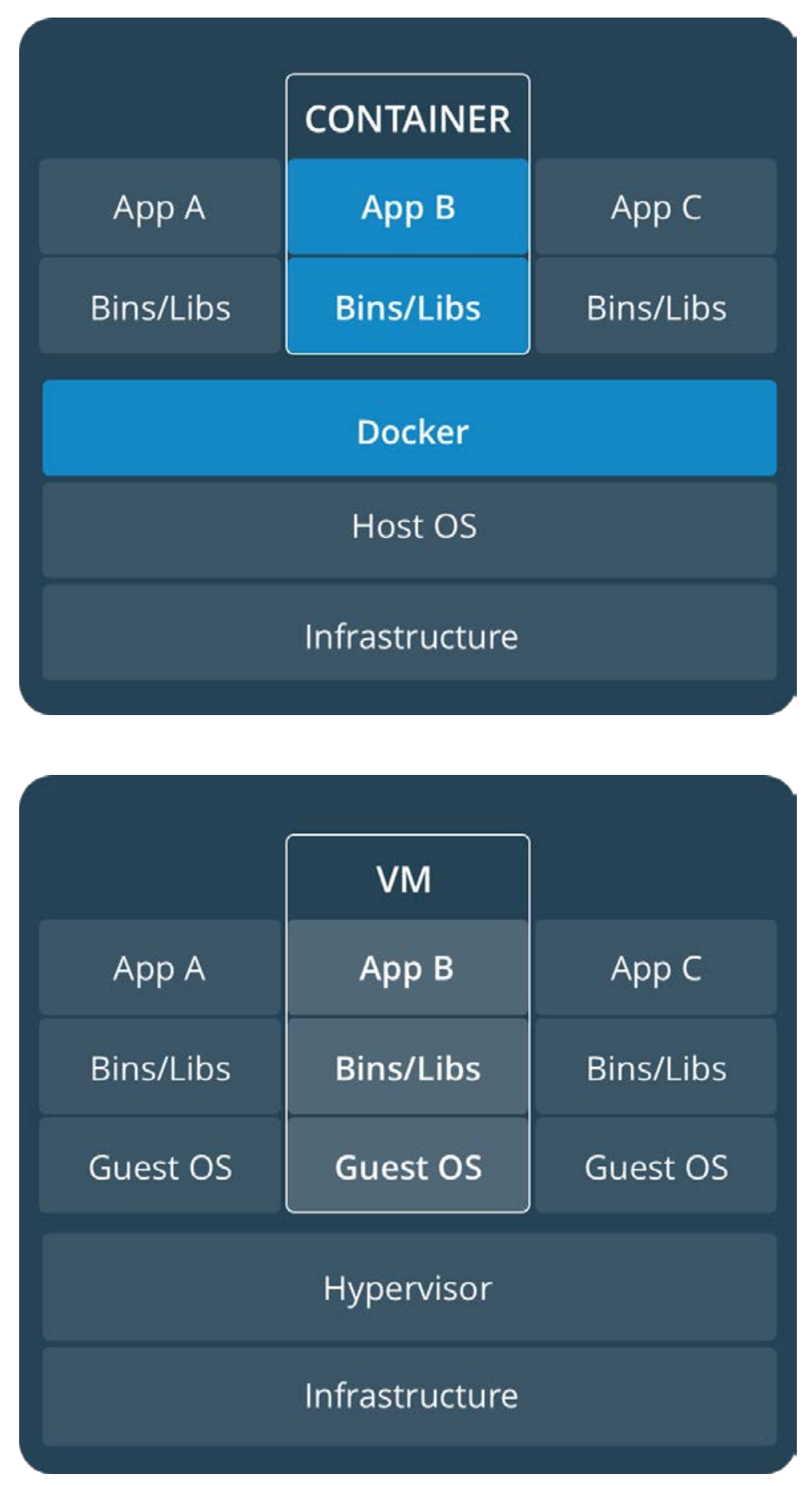


\section{How we run on CORI}

- Submit to CORI like a giant Rubix Cube

- Each compute node has a number of threads that each run a separate instance of the macro

- Each macro runs over all bins few FC points in each bin

- Scale by requesting a large number of nodes

- Wait in regular queue: $\sim 3$ days for big jobs

- Make a reservation: instant access to requested resources

Bins

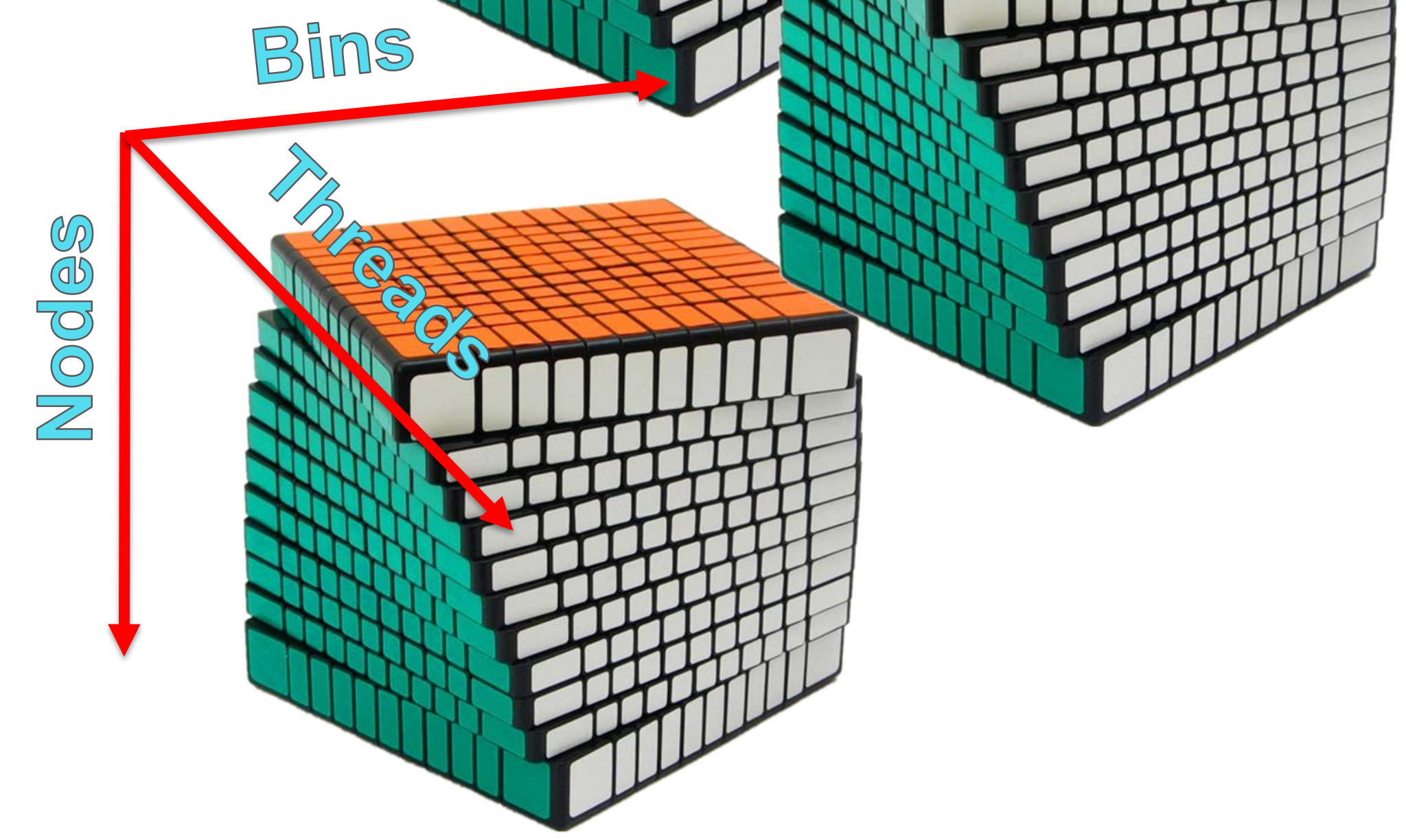




\section{Inverted Hierarchy Rejection}

- $H_{0}: \Delta m_{32}^{2}<0$

- Need a distribution where this is true

- Profile out dependencies on the value of $\Delta m_{32}^{2}$

- Let $\Delta \chi^{2}=0$ if $\vec{\theta}$ is in Inverted Hierarchy

\section{Observed $\Delta \chi^{2} \quad$ Significance}

$$
2.47
$$

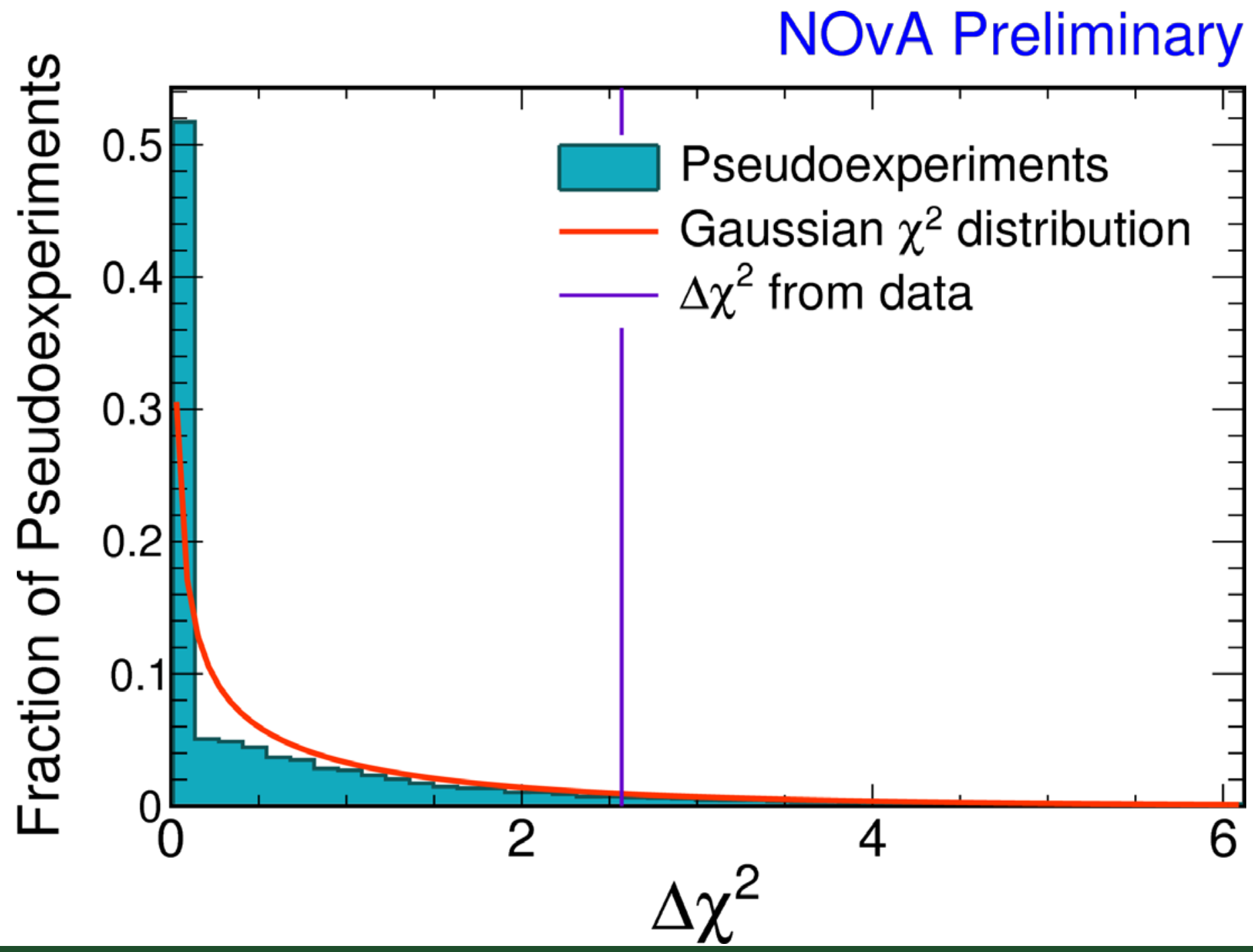

